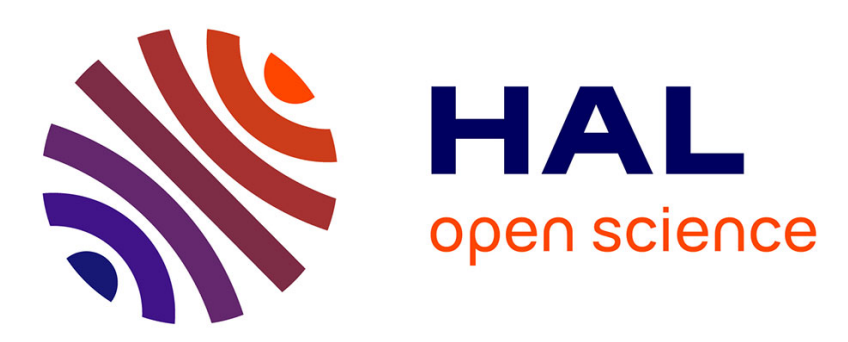

\title{
Diodes Schottky et MIS tunnel sur silicium amorphe hydrogéné de qualité photovoltaïque préparé par pulvérisation cathodique Caractérisation électrique par mesures capacitives
}

\author{
P. Viktorovitch, D. Jousse, A. Chenevas-Paule, L. Vieux-Rochas
}

\section{To cite this version:}

P. Viktorovitch, D. Jousse, A. Chenevas-Paule, L. Vieux-Rochas. Diodes Schottky et MIS tunnel sur silicium amorphe hydrogéné de qualité photovoltaïque préparé par pulvérisation cathodique Caractérisation électrique par mesures capacitives. Revue de Physique Appliquée, 1979, 14 (1), pp.201-208. 10.1051/rphysap:01979001401020100 . jpa-00244577

\section{HAL Id: jpa-00244577 https://hal.science/jpa-00244577}

Submitted on 1 Jan 1979

HAL is a multi-disciplinary open access archive for the deposit and dissemination of scientific research documents, whether they are published or not. The documents may come from teaching and research institutions in France or abroad, or from public or private research centers.
L'archive ouverte pluridisciplinaire HAL, est destinée au dépôt et à la diffusion de documents scientifiques de niveau recherche, publiés ou non, émanant des établissements d'enseignement et de recherche français ou étrangers, des laboratoires publics ou privés. 


\title{
Diodes Schottky et MIS tunnel sur silicium amorphe hydrogéné de qualité photovoltaïque préparé par pulvérisation cathodique Caractérisation électrique par mesures capacitives
}

\author{
P. Viktorovitch, D. Jousse \\ Laboratoire Physique des Composants à Semiconducteurs $\left({ }^{*}\right)$ \\ ENSER, 23, av. des Martyrs, 38031 Grenoble Cedex, France \\ et A. Chenevas-Paule, L. Vieux-Rochas \\ LETI, Commissariat à l'Energie Atomique, CEN/G LETI-EPA \\ 85 X, 38041 Grenoble Cedex, France \\ (Reçu le 26 juin 1978, révisé le 2 octobre 1978, accepté le 2 octobre 1978)
}

\begin{abstract}
Résumé. - Des diodes Schottky et MIS tunnel (Métal-Isolant-Semiconducteur) ont été réalisées sur silicium amorphe hydrogéné obtenu par pulvérisation cathodique. Le matériau présente une forte photoconductivité ; la tension de circuit ouvert et le courant de court-circuit de la diode Schottky sont respectivement $700 \mathrm{mV}$ et $4 \mathrm{~mA} / \mathrm{cm}^{2}$ sous éclairement AM1 en l'absence de couche anti-réfléchissante. Les dispositifs sont utilisés pour la caractérisation électrique du matériau : on relève les caractéristiques courant-tension en fonction de la température et capacité-tension en fonction de la fréquence. On donne une estimation de la distribution des états électroniques dans la bande d'énergie interdite du matériau ; les résultats indiquent une très faible densité d'états autour du milieu de la bande interdite $\left(<10^{16} \mathrm{~cm}^{-3} \mathrm{eV}^{-1}\right)$. Les auteurs mettent en relief les principaux aspects et les difficultés des mesures de capacité de diodes Schottky préparées sur silicium amorphe hydrogéné.
\end{abstract}

\begin{abstract}
Schottky barrier and MIS tunnel diodes are achieved on sputtered hydrogenated amorphous silicon of large photoconductivity; the open-circuit voltage and the short-circuit current of the Schottky diode are respectively $700 \mathrm{mV}$ and $4 \mathrm{~mA} / \mathrm{cm}^{2}$ under AM1 without A.R. coating. The devices are used to carry out electrical characterization of the material. Current-voltage characteristics versus temperature and capacitance-voltage experiments versus frequency are made. An estimation of the electronic states distribution in the energy gap of the material is given; the results indicate a very low density of states around the midgap $\left(<10^{16} \mathrm{~cm}^{-3} \mathrm{eV}^{-1}\right)$. The authors point out the main features and difficulties of capacitance measurements of Schottky diode prepared on hydrogenated amorphous silicon.
\end{abstract}

Le silicium amorphe hydrogéné préparé par pulvérisation cathodique est un matériau très prometteur pour la conversion photovoltaïque de l'énergie solaire [1]. Nous avons réalisé selon cette technique un matériau très photosensible. Nous avons, en outre, élaboré sur ce matériau des diodes Schottky et des structures MIS tunnel en vue de sa caractérisation électrique.

La caractérisation électrique consiste pour l'essentiel en des mesures capacité-tension des diodes en fonction de la fréquence dans le but de déterminer la distribution des états électroniques dans la bande interdite du matériau. La connaissance de cette distribution d'états est très importante pour la compréhension des propriétés électriques et physiques du silicium amorphe hydrogéné. Rappelons qu'un autre

(*) E.R.A., C.N.R.S., nº 659 . moyen d'investigation utilisant l'effet de champ a été développé par Madan et al. [2] pour déterminer la distribution d'états dans le cas du silicium amorphe hydrogéné obtenu par décomposition de silane.

Avant d'aborder la présentation des résultats expérimentaux et de leur interprétation théorique $(\S 2)$, nous décrivons tout d'abord la préparation du matériau et des diodes Schottky $(\S 1)$. Un troisième paragraphe enfin sera consacré aux structures MIS tunnel.

\section{Préparation du matériau et des diodes Schottky.-} Le matériau a été obtenu par pulvérisation cathodique d'une cible de silicium monocristallin (orientation 111, résistivité de type $\mathrm{n} 20 \Omega \mathrm{cm}$; tirage $\mathrm{Cz}$ ) en atmosphère argon/hydrogène. Les paramètres de préparation utilisés sont les suivants :

$$
\begin{aligned}
& P_{\mathrm{H}_{2}}=5 \times 10^{-3} \text { torr } \\
& P_{\text {totale }}=10^{-2} \text { torr }
\end{aligned}
$$


Température du substrat $=300^{\circ} \mathrm{C}$

Vitesse de dépôt $=200 \AA \mathrm{min} .^{-1}$.

Le matériau obtenu contient $15 \%$ atomique d'hydrogène (dosé par réaction nucléaire à l'azote 15) $\left(^{1}\right)$.

La photoconductivité de ce matériau, mesurée en lumière monochromatique (à $2 \mathrm{eV}$ et $10^{15}$ photons $\mathrm{cm}^{-2} \mathrm{~s}^{-1}$ ) atteint $10^{-5} \Omega^{-1} \mathrm{~cm}^{-1}$, ce qui correspond à un produit $\mu \tau$ de $10^{-4} \mathrm{~cm}^{2} \mathrm{~V}^{-1}$, valeur très importante comparée à celles rapportées dans la littérature sur le silicium amorphe obtenu par décomposition de silane [3].

La barrière Schottky a été faite par pulvérisation cathodique diode continue de platine $(80 \AA)$ sur $1 \mu$ de silicium amorphe n'ayant subi aucun traitement de surface mais seulement une remise à l'air entre les deux dépôts. Les conditions du dépôt sont les suivantes :

$P_{\mathrm{Ar}}=3 \times 10^{-2}$ torr

Température du substrat $=100^{\circ} \mathrm{C}$

Tension anode-cathode $\simeq 2000 \mathrm{~V}$.

Le contact arrière est réalisé par une couche évaporée de chrome-antimoine. La structure de la diode est représentée sur la figure 2.

Nous avons observé que les caractéristiques électriques des diodes ne sont pas stables en particulier lorsqu'on les expose à la lumière [4]. C'est pourquoi les mesures capacitives doivent être effectuées en obscurité après un recuit systématique du dispositif au-dessus de $150^{\circ} \mathrm{C}$.

2. Diode Schottky : résultats expérimentaux et interprétation. - 2.1 PROPRIÉTÉS PHOTOÉLECTRIQUES. - 2.1.1 Réponse spectrale. - La figure 1 représente le photocourant de court-circuit (normalisé par rap-

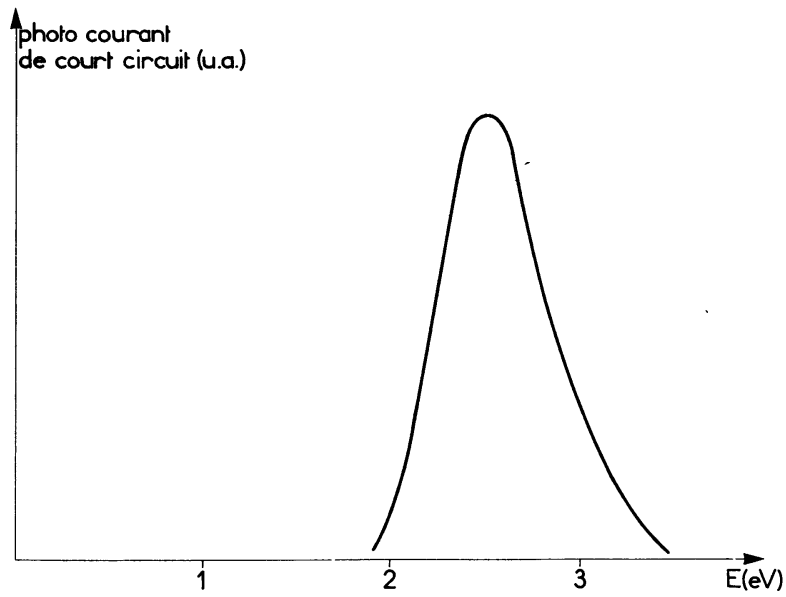

Fig. 1. - Réponse spectrale normalisée de la diode Schottky.

[Normalized spectral response of the cell.]

( ${ }^{1}$ Le dosage a été réalisé par J. P. Thomas de l'IPN de Lyon. port au flux énergétique) de la diode en fonction de l'énergie des photons incidents. Comme le confirment par ailleurs des mesures optiques, la largeur de la bande interdite est de l'ordre de $2 \mathrm{eV}$.

2.1.2 Performances photovoltaïques. - Les caractéristiques photovoltaïques de la cellule sous éclairement AM1 sont représentées sur la figure 2. Le rendement de conversion atteint $1,3 \%$ sans couche anti-reflet. Le courant de court-circuit est d'environ $4 \mathrm{~mA} \mathrm{~cm}^{-2}$ et la tension de circuit ouvert de $700 \mathrm{mV}$. Ces résultats sont à notre connaissance les meilleurs obtenus à ce jour sur du matériau préparé par pulvérisation cathodique, bien qu'étant encore nettement en deçà de ceux obtenus sur du matériau préparé par décomposition de silane (rendement de l'ordre de $5 \%[5,6])$.

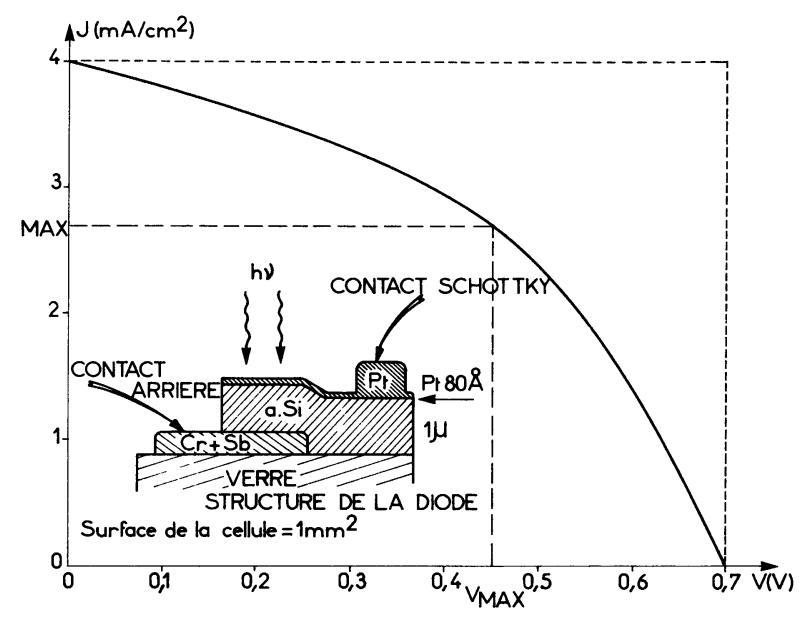

Fig. 2. - Caractéristique courant-tension sous illumination (AM1).

[Current-voltage characteristic under AM1 illumination.]

2.2 CARACTÉRISATION ÉleCtRIQUE. - Nous avons relevé les caractéristiques courant-tension des diodes en fonction de la température dans la gamme : $-85^{\circ} \mathrm{C}<T<180^{\circ} \mathrm{C}$. De plus, des mesures de capacité et de conductance en fonction de la polarisation ont été menées à diverses fréquences à l'aide d'un amplificateur à détection synchrone.

2.2.1 Caractéristiques $I(V)$. - L'exploitation d'un réseau de caractéristique $I(V)$ en fonction de la température nous permet de déduire divers paramètres électriques de la structure. C'est ainsi que la barrière $\varphi_{\mathrm{B}}$ que doivent franchir les électrons du métal pour atteindre la bande de conduction du semiconducteur n'est autre que l'énergie d'activation de l'ordonnée à l'origine de la partie linéaire de la caractéristique directe $\log I(V)$ [7]. Nous trouvons $\varphi_{\mathbf{B}}=0,95 \mathrm{eV}$. Par ailleurs, l'énergie d'activation de la zone ohmique de la caractéristique directe qui est de $0,5 \mathrm{eV}$ traduit approximativement la différence d'énergie moyenne entre la bande de conduction et le quasi-niveau de Fermi des électrons dans les conditions de bandes 
plates. Il est à noter que cette quantité ajoutée à la tension de bandes plates qui est de l'ordre de $0,45 \mathrm{~V}$ donne de nouveau $0,95 \mathrm{eV}$, c'est-à-dire la valeur de $\varphi_{\mathrm{B}}$. Ces divers paramètres électriques sont utiles à l'exploitation des mesures capacitives (voir $\S 2.2 .2$ ).

L'énergie d'activation du courant inverse pour $V=-1 \mathrm{~V}$ est aussi égale à $0,95 \mathrm{eV}$ pour des températures supérieures à $150^{\circ} \mathrm{C}$ (Fig. $3 a$ ). Dans cette gamme de température c'est donc l'émission d'électrons du métal vers la bande de conduction du semiconducteur qui contrôle le transport à travers la structure. Pour des températures inférieures à $80^{\circ} \mathrm{C}$, on atteint un nouveau régime correspondant à une énergie d'activation de l'ordre de $0,2 \mathrm{eV}$. Ce régime de faible énergie d'activation traduit probablement un processus de conduction par sauts dans le matériau. D'ailleurs, nous observons aussi (Fig. $3 b$ ) une énergie d'activation du même ordre de grandeur $(0,15 \mathrm{eV})$ pour la conductance en régime ohmique $G$ (pente de la caractéristique $I(V)$ dans la région ohmique) mais à des températures beaucoup plus faibles $\left(T<-60^{\circ} \mathrm{C}\right)$. Ce comportement en fonction de la température s'explique simplement en remarquant que la jonction présente une barrière beaucoup plus importante aux porteurs transitant par la bande de conduction du semiconducteur qu'à ceux assurant la conduction par sauts en particulier autour du niveau de Fermi, $\mathrm{du}$ moins en polarisation inverse ou pour des faibles

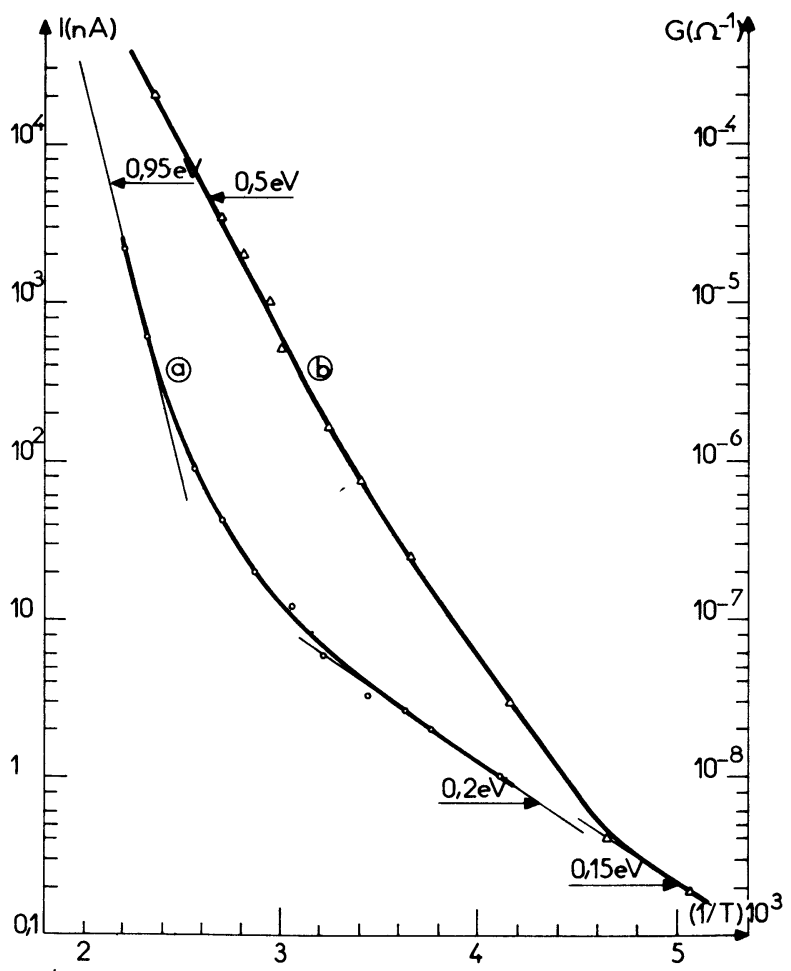

Fig. 3. - Evolutions en fonction de la température du courant inverse pour $V=-1 \mathrm{~V}$ (a) et de la conductance de la diode dans la région ohmique (b).

[Variations versus temperature of the reverse current for $V=-1 \mathrm{~V}$ (a) and of the conductance of the diode in the ohmic region (b).] polarisations directes. Ce n'est évidemment plus le cas dans la zone ohmique, la barrière n'existant plus. Il est maintenant clair que la conduction par sauts est observable à des températures nettement supérieures en polarisation inverse que dans la zone ohmique.

En outre, la conduction par sauts se traduit en direct par un fort coefficient d'idéalité (excédant 2 et pouvant atteindre 6 à 10). Le coefficient d'idéalité demeure important pour des polarisations directes d'autant plus grandes que la température est plus faible, la conduction par sauts étant favorisée. Le coefficient d'idéalité est un bon indicateur de l'importance de la conduction par sauts et donc de celle de la densité de défauts dans le matériau.

Signalons enfin qu'une analyse, à la température ordinaire, du comportement en fonction de la fréquence de la conductance $G$ de la diode permet de confirmer ces hypothèses. Nous observons, en effet, une forte dispersion de $G$ en fonction de la fréquence en polarisation inverse ou pour de faibles polarisations directes; $G$ obéit alors à une loi en $\omega^{(s)}$ avec $s$ très voisin de l'unité, caractéristique de la conduction par sauts [8] (Fig. 4). Au contraire, pour des polarisations directes croissantes, la conductance devient indépendante de la fréquence à la température ordinaire, le transport du courant étant assuré dans ces condi-

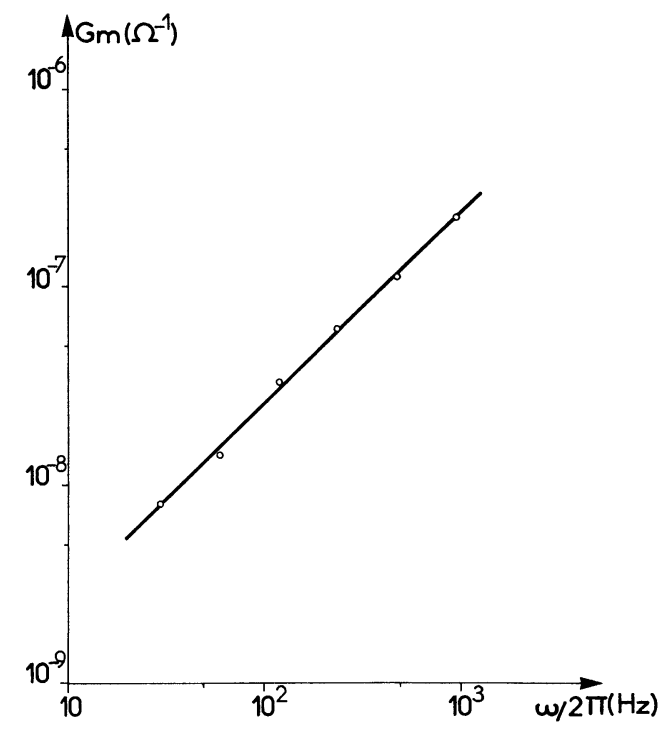

Fig. 4. - Conductance de la diode en fonction de la fréquence à la température ambiante et en l'absence de polarisation continue.

[Conductance of the diode versus frequency for $V=0$ and $T=295 \mathrm{~K}$.]

tions par les électrons libres pour l'essentiel. Bien qu'il semble encore prématuré de définir l'itinéraire emprunté par le processus de conduction par sauts, il est probable que cette conduction soit assurée par des électrons dans la queue de bande de conduction [3] en direct et par des trous dans la queue de bande de valence [3] en inverse. 
2.2.2 Caractéristiques $C(V)$ en fonction de la fréquence. - La figure 5 donne le résultat des mesures capacité-tension en fonction de la fréquence à la température ordinaire. Les expériences ont toutes été effectuées sur le même type de matériau dont les conditions de préparation ont été décrites au paragraphe 1 ; plusieurs dispositifs ont été testés, donnant approximativement des résultats identiques. En raison de la forte résistivité du matériau (supérieure à $10^{9} \Omega \mathrm{cm}$ ) les expériences doivent être effectuées à des fréquences inférieures à $500 \mathrm{~Hz}$, valeur correspondant approximativement à la fréquence de relaxation diélectrique $\mathrm{du}$ matériau. Au-dessus de $500 \mathrm{~Hz}$ nous obtenons simplement la capacité inter-électrodes de $120 \mathrm{pF}$ environ. La capacité continuant d'augmenter en dessous de $5 \mathrm{~Hz}$, nous avons travaillé jusqu'à la fréquence de $1 \mathrm{~Hz}$. Il apparaît que la capacité varie peu en fonction de la tension et de la fréquence en dessous de $0,3 \mathrm{~V}$ puis augmente fortement au-delà et passe par un maximum à $0,65 \mathrm{~V}$.

Nous analyserons ces résultats en considérant successivement les trois situations : $V=0$, polarisation directe et inverse.

1) $\mathrm{A} V=0$, c'est-à-dire autour de l'équilibre nous observons que la capacité varie très lentement avec la fréquence : de $120 \mathrm{pF}$ pour $120 \mathrm{~Hz}$ à $260 \mathrm{pF}$ pour $1 \mathrm{~Hz}$. On peut déduire de ce comportement en fonction de la fréquence que l'extension de la zone de charge d'espace occupe au minimum une épaisseur égale à celle de la couche $(d=1 \mu)$ diminuée d'une longueur de Debye $L$ [9] : il s'agit, bien entendu, de la longueur de Debye associée aux états électroniques localisés dans la bande interdite (voir l'annexe $\mathrm{A}$ ). La légère augmentation de la capacité provient alors de la contribution des états situés autour du niveau de Fermi, qui parviennent à suivre progressivement la modulation lorsqu'on abaisse la fréquence. En effet, le temps de réponse de ces états augmente lorsque nous approchons du métal puisque le niveau de Fermi s'éloigne de la bande de conduction [9] (voir Fig. A.1 de l'annexe). Il est désormais possible de calculer une limite supérieure de la densité moyenne d'états localisés autour du niveau intrinsèque $E_{\mathrm{i}}$ dans le volume du semiconducteur ou, plus précisément, dans une gamme d'énergie de l'ordre de $0,5 \mathrm{eV}$ au-dessus de $E_{\mathrm{i}}$ (voir à ce propos la figure A.1 de l'annexe : le niveau de Fermi en surface est sensiblement au milieu de la bande interdite et le potentiel de surface $V_{\mathrm{s}}$ est de l'ordre de $0,5 \mathrm{~V}$ ). Pour ce faire nous écrivons que l'épaisseur théorique $W_{\mathrm{t}}$ de la zone de charge d'espace, calculée en supposant le milieu semi-infini (voir annexe), est supérieure ou égale à $d-L$. Cette démarche peut paraître artificielle puisque nous venons de voir que le comportement en fonction de la fréquence de la capacité montre que l'espace interélectrode ne peut être considéré comme semi-infini vis-à-vis de l'extension de la zone de charge d'espace sous le contact Schottky. Cependant, en adoptant un raisonnement par l'absurde, il est clair que la condition $W_{\mathrm{t}}<d-L$ ne tient pas, puisqu'elle signifierait que l'espace interélectrode pourrait être considéré comme semi-infini, ce qui est contraire à nos conclusions, déduites des résultats expérimentaux. De la condition $W_{\mathrm{t}} \geqslant d-L$, on déduit :

$$
L\left(\log \frac{q\left|V_{\mathrm{s}_{0}}\right|}{k T}+1\right) \geqslant d .
$$

Cette inégalité fournit une limite supérieure de la densité moyenne d'états (dans une gamme d'énergie d'environ $0,5 \mathrm{eV}$ au-dessus de $E_{\mathrm{i}}$ ) de l'ordre de $10^{22} \mathrm{~m}^{-3} \mathrm{eV}^{-1}$, si l'on prend les valeurs numériques suivantes :

$d=1 \mu ; \quad\left|V_{\mathrm{s}_{0}}\right|=0,5 \mathrm{eV} \quad$ et $\varepsilon=10^{-10} \mathrm{Fm}^{-1}$.

Cette densité d'états très faible explique la forte photoconductivité du matériau (cf. $\S 1$ ) et le bon comportement photovoltaïque du dispositif, notamment la valeur du courant de court-circuit obtenu sans couche anti-reflet. En outre, il est à noter que, bien que les mesures $C(V)$ soient plus difficiles sur silicium amorphe intrinsèque que sur silicium amorphe dopé (mesures basse fréquence), il est nécessaire d'utiliser un matériau intrinsèque pour parvenir à mesurer une densité d'états inférieure à $10^{22} \mathrm{~m}^{-3} \mathrm{eV}^{-1}$; dans un matériau dopé (dopage supérieur à $10^{22} \mathrm{~m}^{-3}$ ), la capacité est en réalité imposée par le dopage.

2) En polarisation inverse, la capacité varie très peu avec la fréquence et décroît très lentement vers la capacité interélectrode lorsque l'on polarise la diode plus fortement en inverse. Ceci est dû au fait que, en inverse, la zone de charge d'espace est hors d'équilibre et en régime de désertion; l'occupation des états est donc de moins en moins contrôlée par les porteurs libres et, en conséquence, répond de plus en plus difficilement à la modulation de potentiel ce qui conduit à une diminution de la capacité.

3) En polarisation directe, la capacité croît brutalement vers $0,4 \mathrm{~V}$, notamment à basse fréquence. L'augmentation de la capacité mesurée est due à l'accroissement de la densité d'états localisés près de la bande de conduction dans le semiconducteur. $\mathrm{Si}$ l'on admet que l'occupation de ces états est contrôlée par le quasi-niveau de Fermi des électrons libres (ce qui est une hypothèse raisonnable en direct) leur contribution à la capacité s'accroît dans la mesure où, sous la polarisation directe appliquée, le niveau de Fermi des électrons se rapproche de la bande de conduction.

Il semble que la contribution des états de surface soit négligeable : ils conduiraient à une valeur de capacité dépendant plus fortement de la fréquence et à un pic sur les courbes de conductance en fonction de la tension appliquée, ce qui n'a pas été observé [10]. On peut donc conclure que, soit la densité d'états de surface est négligeable, soit leur taux d'occupation est contrôlé par le niveau de Fermi du métal, ces deux 
propositions ne s'excluant pas mutuellement. Il est par ailleurs aisé de vérifier que la contribution à la capacité totale mesurée des électrons libres s'accumulant, en polarisation directe, à la surface de la diode est négligeable; un calcul simple montre que cette contribution est au moins cinquante fois inférieure à la valeur mesurée à $1 \mathrm{~Hz}$ jusqu'à une tension appliquée de 0,5 à $0,6 \mathrm{~V}$.

On peut montrer [9] que le maximum de la caractéristique $C(V)$ pour $V=0,65 \mathrm{~V}$ est dû au fait que la résistance de contact (qui contrôle le courant thermo-ionique) devient inférieure à la résistance du volume du matériau (qui contrôle la diffusion des électrons à travers la zone de charge d'espace). Cet effet est d'autant plus marqué que le transport des porteurs à travers la jonction est contrôlé par leur diffusion à travers la zone de charge d'espace [11] plutôt que par leur émission thermo-ionique. Il est à noter que la théorie thermo-ionique n'est pas rigoureusement applicable dans le cas d'une diode Schottky pure sur silicium amorphe sans couche isolante intermédiaire [9], en raison de la faible mobilité des électrons [7,12]. Pour les tensions supérieures à $0,65 \mathrm{~V}$, la capacité tend vers la capacité interélectrode en l'absence de contribution du contact arrière.

Notons l'apparition d'un maximum secondaire sur la caractéristique $C(V)$ à $30 \mathrm{~Hz}$ et pour $V=0,4 \mathrm{~V}$ en direct (Fig. 5). Ce maximum disparaît à basse fréquence. Cet effet est encore plus net sur les caractéristiques $C(V)$ de la figure 6 obtenues sur une autre diode Schottky. Ce maximum indique vraisembla-

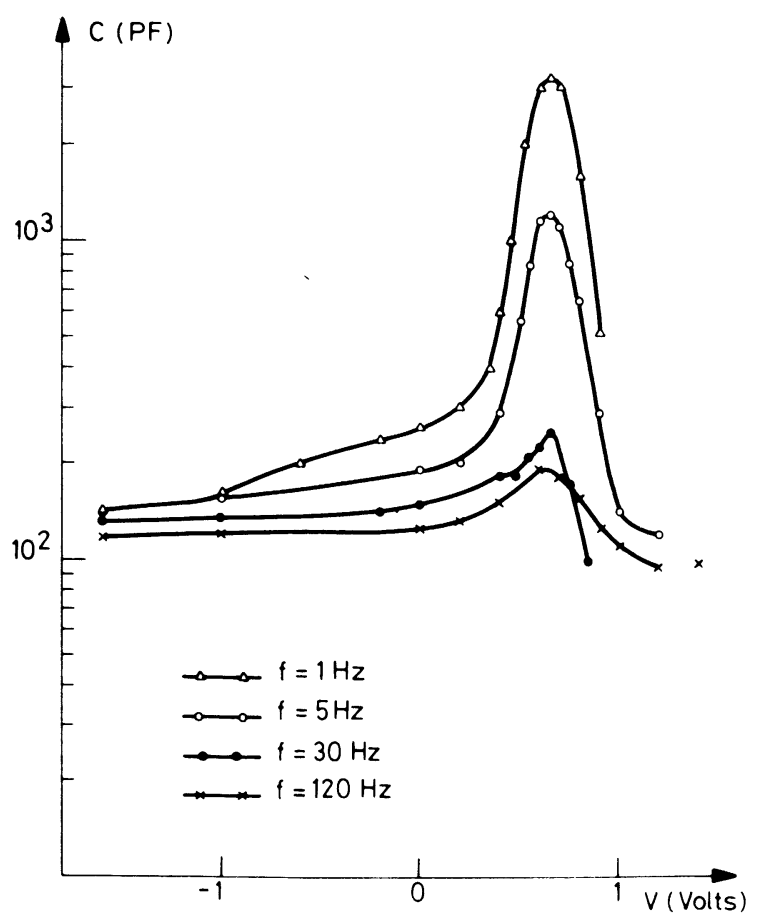

Fig. 5. - Caractéristiques $C(V)$ de la diode Schottky pour diverses fréquences et à la température ordinaire.

$[C(V)$ characteristics of the Schottky diode at various frequencies and at ordinary temperature.]

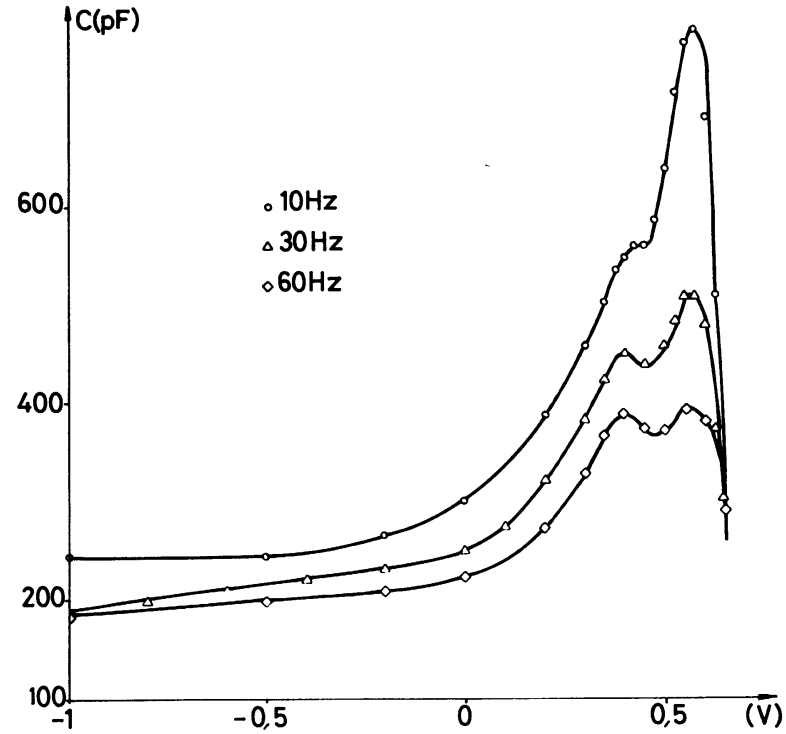

Fig. 6. - Caractéristiques $C(V)$ pour trois fréquences. On observe un maximum secondaire.

$[C(V)$ characteristics at three frequencies. A secondary maximum is observed.]

blement l'existence d'un pic d'états rapides situés à environ $0,5 \mathrm{eV}$ de la bande de conduction. Un tel pic a déjà été observé à $0,4 \mathrm{eV}$ de la bande de conduction sur du silicium amorphe préparé par décomposition de silane par Madan et al. [2] en utilisant la mesure d'effet de champ. La disparition de ce maximum à basse fréquence indique la présence d'états lents dont la contribution devient plus importante lorsque l'on diminue la fréquence de modulation au point de masquer l'effet des états plus rapides. Remarquons que cette analyse en fréquence nous permet de réaliser une spectroscopie des états.

A partir de la caractéristique $C(V)$ à $1 \mathrm{~Hz}$ (Fig. 5), nous pouvons déduire une estimation des variations de la densité d'états $D(E)\left(\mathrm{m}^{-3} \mathrm{eV}^{-1}\right)$ où $\mathrm{E}$ est l'énergie comptée à partir de la bande de conduction; nous avons [9] :

$D(E)=\frac{1}{q \varepsilon}\left(C_{\mathrm{t}}^{2}-Q_{\mathrm{t}} \frac{\mathrm{d} C_{\mathrm{t}}}{\mathrm{d} V}\right)$

où $C_{\mathrm{t}}$ et $Q_{\mathrm{t}}$ sont la capacité et la charge d'espace correspondant au potentiel pour lequel le quasi-niveau de Fermi des électrons est situé à $E$ en dessous de la bande de conduction à la surface du semiconducteur. La relation (1) est obtenue avec les hypothèses suivantes : d'une part, la fréquence est suffisamment basse pour que l'on puisse négliger les résistances série (comprenant les résistances fictives représentant la cinétique des états) devant les capacités correspondantes; d'autre part, la résistance de contact est beaucoup plus grande que les résistances série (on considère la région $V<0,6 \mathrm{eV}$ ); enfin, on applique la théorie thermo-ionique ce qui, comme nous l'avons 
déjà signalé, constitue une approximation. La figure 7 montre la courbe $D(E)$ pour $E$ compris entre $0,95 \mathrm{eV}$ et $0,35 \mathrm{eV}$. La densité $D(E)$ augmente brutalement à $0,55 \mathrm{eV}$ de la bande de conduction; notre estimation donne une densité d'états très faible autour du milieu de la bande interdite.

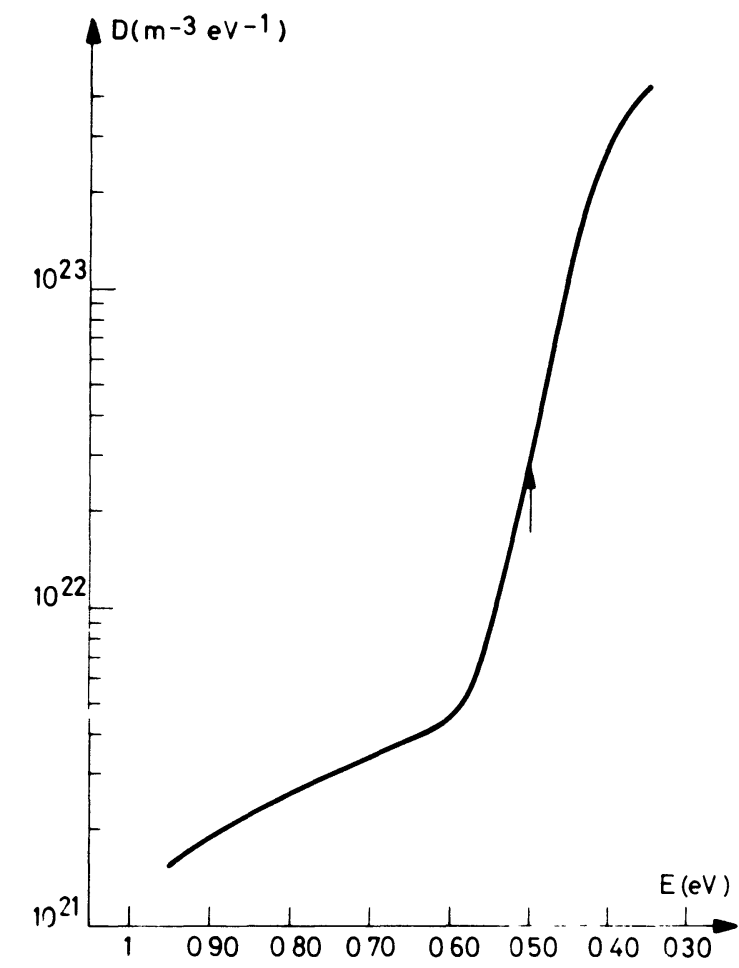

Fig. 7. - Allure de la densité d'états localisés dans la bande interdite du silicium amorphe en fonction de l'énergie $E$ comptée à partir du bord de la bande de conduction. La flèche indique les conditions de bandes plates.

[Shape of the density of localized states distribution in the gap of the amorphous silicon versus the energy distance from the conduction band edge. The arrow denotes the flat band conditions.]

3. Structure MIS tunnel. - L'intérêt de la structure MIS pour la conversion photovoltaïque est maintenant bien établi [13-16]. Nous avons également réalisé des structures MIS sur le même silicium amorphe; nous avons pour cela exposé le matériau à une atmosphère oxydante avant le dépôt de la couche métallique. Nous ne sommes cependant pas encore en mesure de contrôler l'épaisseur de la couche isolante. C'est pourquoi nous avons obtenu des dispositifs peu performants pour la conversion photovoltaïque; leur caractéristique $I(V)$ sous illumination rend compte d'un faible courant de court-circuit et présente deux points d'inflexion, dont un est situé autour de l'axe des tensions, ce qui est la manifestation d'une couche isolante trop épaisse $[15,16]$.

Il apparaît, en outre, que la structure MIS tunnel est aussi mieux adaptée pour la caractérisation du silicium amorphe par mesures capacitives [9]. En effet, en présence d'une couche isolante intermédiaire entre le métal et le semiconducteur, la vitesse de collection des électrons par le métal est considérablement réduite ce qui renforce la validité de l'approximation thermoionique. Dans ces conditions, le maximum principal observé sur les caractéristiques $C(V)$ relevées sur diodes Schottky est déplacé dans le cas des structures MIS vers des polarisations directes plus élevées ou peut même éventuellement disparaître. C'est ce que nous observons sur les caractéristiques $C(V)$ de la figure 8 relevées sur une diode MIS réalisée sur le même silicium amorphe hydrogéné. La structure MIS devrait donc nous permettre d'explorer une gamme d'énergie dans la bande interdite du semiconducteur plus large qu'avec la diode Schottky.

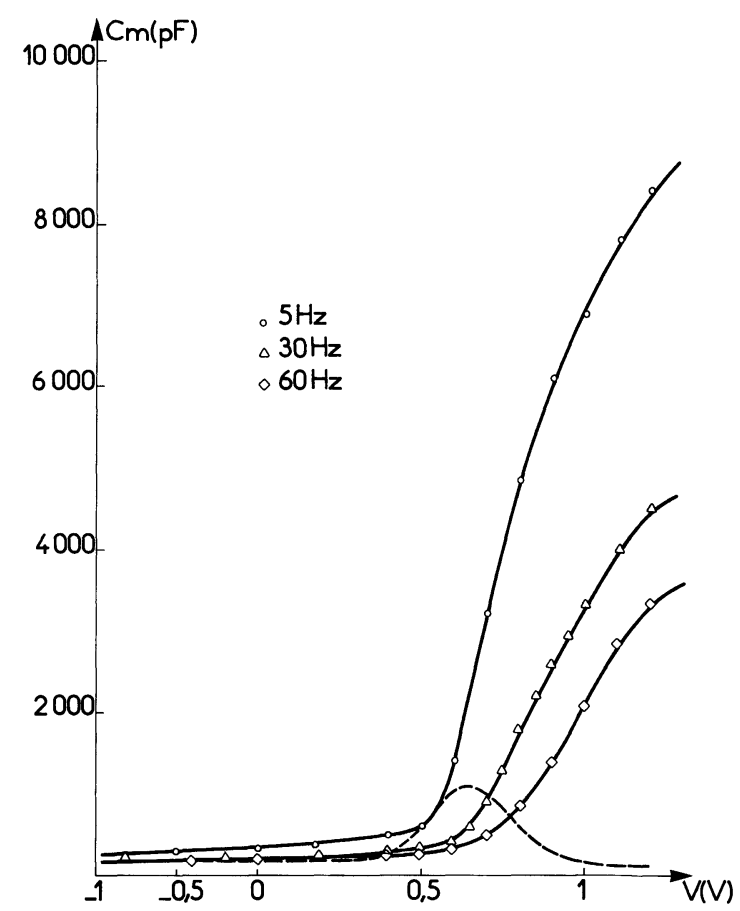

Fig. 8. - Caractéristiques $C(V)$ pour trois fréquences d'une diode MIS sur silicium amorphe hydrogéné. La caractéristique $C(V)$ à $5 \mathrm{~Hz}$ de la diode Schottky (voir Fig. 5) est reportée en trait discontinu.

$[C(V)$ characteristics at three frequencies of a MIS diode on hydrogenated amorphous silicon. The dotted line concerns the $C(V)$ characteristic of the Schottky diode at $5 \mathrm{~Hz}$ (see Fig. 5).]

4. Conclusion. - En résumé nous avons préparé, par la technique de pulvérisation cathodique, du silicium amorphe hydrogéné de bonne qualité en vue d'applications pour la conversion photovoltaïque.

Ce résultat est confirmé par les mesures $C(V)$ sur diode Schottky en fonction de la fréquence, dont l'interprétation indique une très faible densité d'états dans notre matériau. Cette méthode de caractérisation se révèle très prometteuse pour la détermination de la distribution des états localisés dans le silicium amorphe hydrogéné bien que l'interprétation des résultats expérimentaux soit assez délicate sur un tel 
matériau en comparaison du silicium monocristallin. Dans cet article nous avons fait ressortir les principaux aspects et les difficultés de cette méthode.

Les expériences et résultats préliminaires présentés dans cet article démontrent l'efficacité des mesures électriques $C(V)$. Nous nous proposons dans l'avenir d'augmenter cette efficacité en faisant d'étroites corrélations entre la caractérisation électrique (utilisant des diodes MIS et des structures capacitives métal-isolant épais-a $\mathrm{SiH}$ ) et d'autres types de caractérisation physique. Nous envisageons évidemment aussi de suivre l'évolution des caractéristiques électriques des dispositifs en fonction des conditions de préparation du matériau.

Annexe. - Epaisseur $W_{\mathrm{t}}$ DE La zone de Charge D'ESPACE. - Nous nous proposons de calculer l'épaisseur $W_{\mathrm{t}}$ de la zone de charge dans un milieu semi-infini (le volume du matériau est supposé illimité). Nous examinons le cas de l'équilibre (Fig. A.1), c'est-à-dire en l'absence de polarisation appliquée. Si nous négligeons la contribution des porteurs libres, la charge d'espace locale est contrôlée par la distribution des états. Dans le volume neutre, pris comme origine des potentiels, la charge totale des états est globalement nulle (semiconducteur compensé). A l'abscisse $x$ dans la zone de charge d'espace, où le potentiel est $U$, la charge d'espace $\rho(x)$ est due aux états qui ont traversé le niveau de Fermi

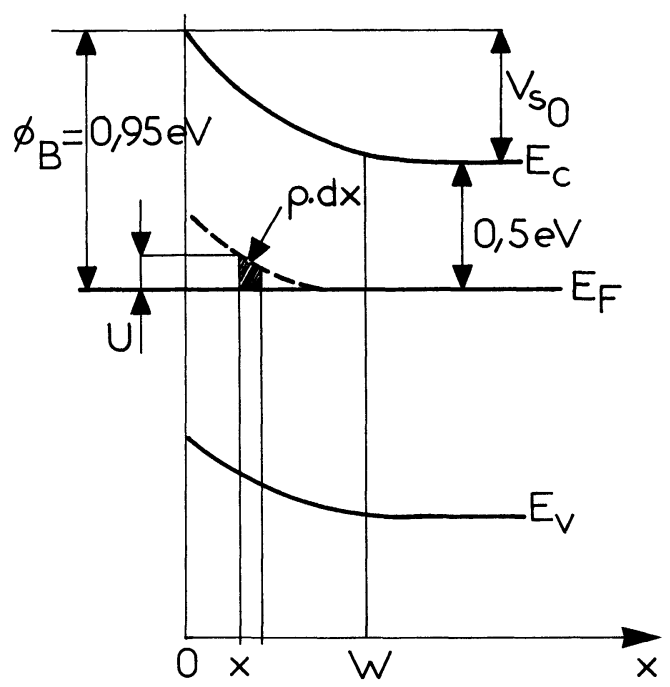

Fig. A.1. - Diagramme de bandes de la jonction à l'équilibre, sans polarisation extérieure.

[Band diagram of the junction at equilibrium, without external applied voltage.] en raison de la courbure des bandes. Si $D^{+}(E)$ et $D^{-}(E)$ sont respectivement les densités d'états de type donneur et de type accepteur $\left(\mathrm{m}^{-3} \mathrm{eV}^{-1}\right), \rho(x)$ s'exprime en fonction de la somme

$D^{+}(E)+D^{-}(E)=D(E)$

où $D(E)$ est la densité totale d'états $\left(\mathrm{m}^{-3} \mathrm{eV}^{-1}\right)$ :

$\rho(x)=q \int_{E_{\mathrm{F}}(x)}^{E_{\mathrm{F} v}} D(E) \mathrm{d} E$

où $E_{\mathrm{F}}(x)$ est le niveau de Fermi (qui est rectiligne et le même pour les électrons libres, les trous et les états, le semiconducteur étant à l'équilibre), $E_{\mathrm{Fv}}$ est le niveau de Fermi en volume compté par rapport au bord de la bande de conduction; $E$ et $E_{\mathrm{F}}(x)$ sont comptés par rapport au bord de la bande de conduction $E_{\mathrm{c}}(x)$ en $x$. Finalement on suppose la température nulle, ce qui est évidemment une approximation.

Dans le cas simple d'une distribution uniforme d'états en fonction de l'énergie, on peut écrire :

$\rho(x)=-q D_{v} U(x)$

où $D_{v}$ est la densité d'états en volume $\left(\mathrm{m}^{-3} \mathrm{eV}^{-1}\right)$, et $q=1,6 \times 10^{-19}$ C. L'équation de Poisson fournit une autre relation entre $U(x)$ et $\rho(x)$ :

$\frac{\mathrm{d}^{2} U}{\mathrm{~d} x^{2}}=-\frac{\rho(x)}{\varepsilon}$

$\varepsilon$ étant la permittivité du semiconducteur.

De (A.2) et (A.3) on déduit simplement :

$U(x)=V_{\mathrm{s}_{0}} \exp \left(-\frac{x}{L}\right)$

où $V_{\mathrm{s}}$ est le potentiel de surface et $L$ la longueur de Debye associée aux états localisés

$L=\left(\frac{\varepsilon}{q D_{v}}\right)^{1 / 2}$.

Si nous définissons la limite entre la zone de charge d'espace et le volume neutre comme correspondant à un potentiel local de $k T / q$, il vient :

$W_{\mathrm{t}}=L \log \frac{q\left|V_{\mathrm{s}_{0}}\right|}{k T}$

$W_{\mathrm{t}}$ est fixée par les états localisés. Notons que pour une température finie, la relation (A.6) n'est plus valable pour $\left|V_{\mathrm{s}_{0}}\right|<k T / q$, en raison de l'approximation de température nulle faite pour exprimer $\rho(x)$. 


\section{Bibliographie}

[1] Paul, W., Lewis, A. J., Connell, G. A. N. et MoustaKAS, T. D., Solid State Commun. 20 (1976) 969.

[2] Madan, A., Le Comber, P. G. et Spear, W. E., J. Non-Cryst. Solids 20 (1976) 239.

[3] SPear, W. E., Adv. Phys. 26 (1977) 811.

[4] Staebler, D. L. et Wronski, C. R., Appl. Phys. Lett. 31 (1977) 292.

[5] Wronski, C. R., Carlson, D. E., Daniel, R. E., Appl. Phys. Lett. 29 (1976) 602.

[6] McGill, J., Wilson, J. I. B. et KinMond, S., à paraître dans Appl. Phys. Lett.

[7] SzE, S. M., Physics of Semiconductor Devices (Wiley, New York) 1969, p. 394.

[8] MotT, N. F. and Davis, E. A., Electronic Processes in Non-
Crystalline Materials (Clarendon Press, Oxford) 1971, p. 51 .

[9] VikToRovitch, P., non publié.

[10] Kar, S., DahlKe, W., Solid State Electron. 15 (1972) 221.

[11] Crowell, C. R. et Sze, S. M., Solid State Electron 9 (1966) 1035.

[12] Moore, A. R., Appl. Phys. Lett. 31 (1977) 762.

[13] Fonash, S. J., J. Appl. Phys. 46 (1975) 1286.

[14] Viktorovitch, P., Kamarinos, G., Even, P., C. R. Hebd. Séan. Acad. Sci. 283B (1976) 119.

[15] Viktorovitch, P., Kamarinos, G., J. Appl. Phys. 48 (1977) 3060.

[16] Pananakakis, G., Viktorovitch, P., Ponpon, J. P., Revue Phys. Appl. 13 (1978) 449. 